\title{
BMJ Open Glasgow Prognostic Score and modified Glasgow Prognostic Score and survival in patients with hepatocellular carcinoma: a meta-analysis
}

\author{
Lingling Lu, ${ }^{1}$ Kunzhe Lin, ${ }^{2}$ Jiaolong Zheng, ${ }^{3}$ Haicong Wu, ${ }^{3}$ Dongliang Li (D) ${ }^{1,3}$
}

To cite: Lu L, Lin K, Zheng J, et al. Glasgow Prognostic Score and modified Glasgow Prognostic Score and survival in patients with hepatocellular carcinoma: a meta-analysis. BMJ Open 2021;11:e053061. doi:10.1136/ bmjopen-2021-053061

- Prepublication history and additional supplemental material for this paper are available online. To view these files, please visit the journal online (http://dx.doi.org/10.1136/ bmjopen-2021-053061).

$\mathrm{LL}$ and $\mathrm{KL}$ contributed equally.

Received 03 May 2021

Accepted 12 December 2021

Check for updates

(c) Author(s) (or their employer(s)) 2021. Re-use permitted under CC BY-NC. No commercial re-use. See rights and permissions. Published by BMJ.

${ }^{1}$ Fuzong Clinical Medical College of Fujian Medical University,

Fuzhou, Fujian, China

${ }^{2}$ Department of Neurosurgery, Affiliated Fuzhou First Hospital of Fujian Medical University, Fuzhou, Fujian, China

${ }^{3}$ Department of Hepatobiliary Disease, 900th Hospital of the People's Liberation Army Joint Logistics Support Force, Fuzhou, Fujian, China

Correspondence to

Dr Dongliang Li;

Idliang900@163.com

\section{ABSTRACT}

Objective To evaluate the association between inflammation-related markers, modified Glasgow Prognostic Score (mGPS) and Glasgow Prognostic Score (GPS), and survival outcome and recurrence risk in patients with hepatocellular carcinoma (HCC) after treatment.

Design Systematic reviews and meta-analysis of cohort studies.

Date sources Embase, Scopus, Web of Science and PubMed were searched through 10 March 2021.

Eligibility criteria We included cohort studies that assessed the effect of pretreatment mGPS/GPS levels on survival outcomes in patients with HCC.

Data extraction and synthesis Two researchers independently selected the data and reached a consensus. In case of disagreement, a third researcher was required to assist. The HRs and 95\% Cls were used as the effect size indexes. Newcastle-Ottawa Scale was used to assess risk of bias and quality assessment of the included studies. Results The meta-analysis included 23 studies, most of which were retrospective. Participants were grouped according to the score of mGPS/GPS. When analysed into two groups (1/2 vs 0 ), the results showed that patients with a mGPS/GPS of 1 or 2 had poorer overall survival $(0 S)$ than those with a score of 0 (both $p<0.001$ ). When analysed into three groups ( 1 vs 0 and 2 vs 0 ), the results revealed that an mGPS/GPS of 2 is related to poorer OS in patients with $\mathrm{HCC}(\mathrm{HR}=2.46,95 \% \mathrm{Cl} 2.06$ to 2.95 , and $\mathrm{HR}=3.45,95 \% \mathrm{Cl} 1.68$ to 7.10 , respectively). However, a GPS of $1(p=0.005)$ but not an mGPS of $1(p=0.177)$ had a significant association with OS. No association was found between mGPS/GPS and disease-free survival or recurrence-free survival.

Conclusion GPS was more closely associated the survival in patients with HCC than mGPS. A higher GPS has an association with poorer survival. It can be combined with tumour staging to assess the OS of HCC more accurately. PROSPERO registration number CRD42021242049.

\section{INTRODUCTION}

Hepatocellular carcinoma (HCC) is a serious public health problem, especially in Asian countries with a high incidence of hepatitis B. It endangers human life and health seriously. The number of deaths associated with HCC

\section{Strengths and limitations of this study}

- This meta-analysis is the first study to combine Glasgow Prognostic Score and modified Glasgow Prognostic Score in patients with liver cancer and to analyse them in specific groups.

- The source of heterogeneity can be reduced when studies with the same cut-off values are grouped for analysis.

- Prospective studies were included in the metaanalysis that could reduce the risk of selection bias.

- There is no guarantee that the HRs extracted from each study were analysed by the same set of covariates.

- The literature included in this study lacked randomised controlled studies.

each year is almost the same as the incidence of HCC. ${ }^{1}$ Liver resection is still the main treatment for liver cancer, and liver transplantation is feasible in advanced stage. However, despite the variety of treatments, there are significant differences in recurrence and long-term survival. ${ }^{2}$

The prognostic factors of HCC have been studied for many years, including tumour, node, metastasis staging, histological type, tumour number, depth of invasion, vascular invasion and distant metastasis. However, in addition to stage and pathological type, other prognostic factors are still controversial. To achieve accurate and individualised treatment, it is urgent to find appropriate markers to assess the survival. Serum clinical trial indicators have the advantages of being non-invasive, simple, economical and reproducible. Therefore, it is very meaningful to find non-invasive and readily achievable variables before treatment to assess the survival outcomes of patients with cancers. As a routine test indicator, $\mathrm{C}$ reactive protein (CRP) and serum albumin are readily available inflammatory factors. Forrest et $a \hat{l}$ used these two indicators in combination 
as a prognostic score for patients with non-small-cell lung cancer. Many studies have confirmed its prognostic value in malignant tumours ${ }^{4-6}$ and have defined it as the Glasgow Prognostic Score (GPS). Depending on the scoring system, the score of GPS is between 0 and 2. However, in malignant tumours, hypoalbuminaemia without increased CRP is rare. Therefore, mGPS was improved on the basis of GPS. ${ }^{7}$ None of these anomalies will be assigned a score of 0 . GPS and mGPS has been proven to be a predictor of the survival of some tumours, including those of the digestive and respiratory systems. ${ }^{3-68}$

Several studies have indicated a relationship between modified Glasgow Prognostic Score (mGPS)/GPS and survival in patients with HCC. ${ }^{9-11}$ However, due to the difference in study design and the limitation of sample size, the results lack consistency, and according to their research, this opinion is still controversial. Moreover, it is debatable which is more appropriate for the prognosis of HCC. Since the measured values of each individual study report may have a certain degree of bias, a meta-analysis was used to study a specific problem. We performed a meta-analysis that pooled existing data to find an association between the level of mGPS/GPS and the survival of patients with HCC.

\section{MATERIAL AND METHODS}

\section{Search strategy}

Systematic searches were conducted in Embase, Scopus, Web of Science and PubMed. The time limit was from the establishment of each database to 10 March 2021. The data reports in this review are consistent with Preferred Reporting Items for Systematic Reviews and Meta-Analyses ${ }^{12}$ statement (online supplemental table S1). Meta-analysis of Observational Studies in Epidemiology guidelines ${ }^{13}$ were used for study design and manuscript preparation (online supplemental table S2). The protocol was registered in the International Prospective Register of Systematic Reviews (registration number: CRD42021242049).

The subject words and free words were used to search comprehensively. The language of publication was limited to English. If the full text was not accessible, the author was contacted. The complete search terms used in this study are listed in online supplemental table S3. The following search terms were used: "Liver Neoplasm", "primary liver carcinoma", "liver carcinoma", "HCC", "Hepatic Cancer", "Cancer of the Liver", "Liver Cell Carcinoma", "Liver Cancer", "Hepatocellular Carcinoma", "Hepatocellular Cancer", "Hepatic Neoplasm", "Hepatoma", "hepatic carcinoma", "hepatic cell carcinoma", "Cancer of Liver", "hepatocarcinoma", "modified Glasgow prognostic score", "Glasgow prognostic score", "GPS" and "mGPS". Moreover, references that have been included in the literature were also traced.

\section{Inclusion and exclusion criteria}

The inclusion criteria were as follows: (1) the diagnosis of HCG was based on pathological examination or currently ongoing clinical guidelines; (2) articles investigated the relationship between GPS/mGPS and the outcome of HCC; (3) CRP and albumin markers were measured prior to treatment; (4) full-text papers were published; and (5) the outcomes included overall survival (OS), recurrencefree survival (RFS) or disease-free survival (DFS). OS was defined as the interval between the beginning of treatment and death of any cause or the end of follow-up, whichever came first. DFS and RFS were defined as the time between the date of treatment to the date of the first tumour recurrence or death (DFS) and the discovery of recurrence at any site (RFS), respectively.

The exclusion criteria were as follows: (1) reviews, commentaries and case reports; (2) animal experimental research; (3) duplicated or overlapped datasets (only the study with the most up-to-date information prevailed); (4) HRs and 95\% CIs were not available; and (5) studies not written in English.

\section{Data collection}

Two researchers (LL and KL) independently selected the data according to the study design and reached a consensus. In case of disagreement, a third researcher was required to assist. The data were recorded in a spreadsheet. The extracted data included the year of publication, first author, treatment, cut-off value, tumour stage, metastasis, follow-up time, sample size, HR and 95\% CI. HRs from multivariate analysis was preferred.

\section{Quality assessment}

The Newcastle-Ottawa Scale (NOS) was used to measure the quality of the included literature and to assess the risk of bias. A high-quality essay must be scored above 5 points. Two researchers (LL and JZ) conducted quality assessments.

\section{Statistical analyses}

All the data were statistically analysed by Stata V.12.0. Cochran's $Q$ test and the Higgins $I^{2}$ statistic $^{14}$ were used to assess the heterogeneity of the included studies. When $\mathrm{I}^{2}$ is greater than $75 \%$, heterogeneity is high; when $\mathrm{I}^{2}$ is $50 \%-75 \%$, heterogeneity is medium; and when $\mathrm{I}^{2}$ is less than $50 \%$, heterogeneity is low. If the $\mathrm{I}^{2}$ was greater than $50 \%$ or $\mathrm{p}<0.05$, indicating significant statistical heterogeneity between studies, a random-effect model was used. Conversely, the fixed-effect model was used. The relationship between mGPS/GPS and OS in HCC was assessed by HR and 95\% CI. For studies with significant heterogeneity, subgroup analysis would be conducted to further explore the source of heterogeneity according to different covariate groupings. Sensitivity analysis assessed the stability of the results by combining the results after the exclusion of one of the studies to determine the impact of the study on the results. Publication bias was evaluated by Begg's funnel plot and Egger's test. When the $p$ value was $<0.05$, publication bias existed, and the trim-and-fill analysis was used to estimate the lack of studies due to publication bias. All statistical tests were 


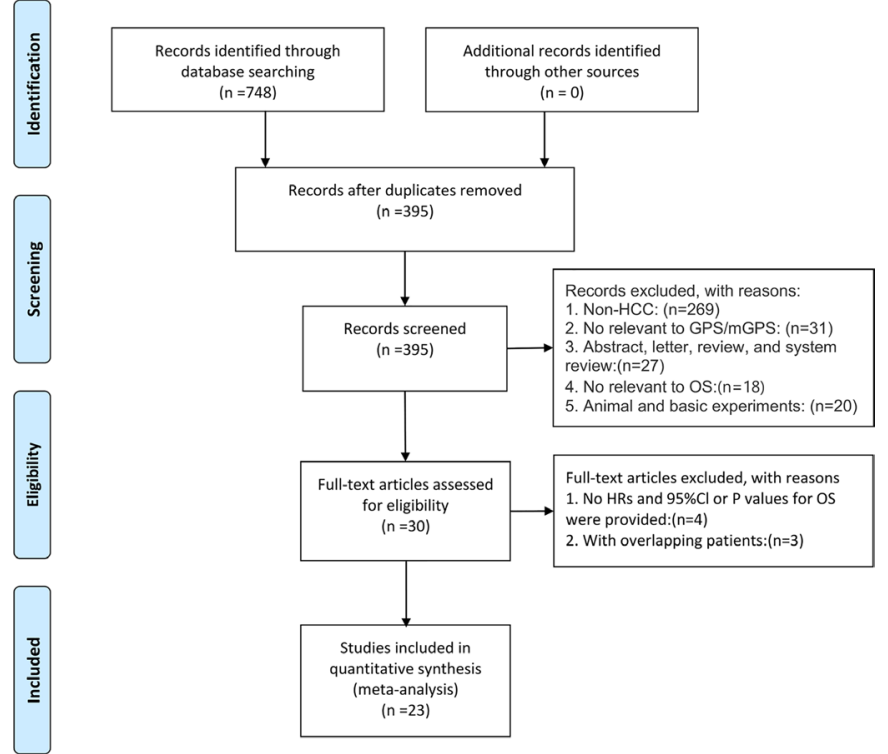

Figure 1 Flowchart of relevant literature screening. GPS, Glasgow Prognostic Score; HCC, hepatocellular carcinoma; mGPS, modified Glasgow Prognostic Score; OS, overall survival.

conducted on two sides, and a p value of $<0.05$ was considered statistically significant.

\section{Patient and public involvement}

Patients or the public were not involved in the design, conduct, reporting or dissemination plans of this research.

\section{RESULTS}

\section{Study selection and basic characteristics}

A total of 748 studies were initially identified, which were retrieved from four databases, and no relevant research was found elsewhere. After deleting duplicate articles, 395 articles were retained. After screening titles and abstracts, 365 articles that did not meet the criteria were excluded. By reading 30 full-text articles, 3 articles with duplicate data and four articles that could not provide effect size were eliminated. Ultimately, 23 studies $^{9-11} 15-34$ were included in the meta-analysis (figure 1). The basic characteristics of 23 studies are presented in table 1. Most studies were performed in Asia, and only two studies were conducted in Europe. The sample size ranges from 81 to 1625 . In terms of treatment methods, patients in 12 studies underwent hepatectomy, and patients in 11 studies underwent other treatment methods, including drug, ablation, radiotherapy and transcatheter arterial chemoembolisation. Regarding the variables of the study, except for two studies that only used univariate analysis for evaluation, the remaining 21 studies directly reported the HR and $95 \%$ CI generated by the multivariate analysis. However, these 21 original studies did not use the same set of covariables in their multivariable analyses. The risk of bias for each study was assessed separately. The NOS score was equal to or greater than 6 in 19 cohorts, which meant relatively high quality (figure 2 ). The NOS score for each study is listed in online supplemental table $\mathrm{S} 4$, and basic features of the included studies are listed in online supplemental table S5.

\section{GPS/mGPS and OS}

There are 18 papers that assessed the relationship between GPS and OS. As mentioned previously, GPS is between 0 and 2. Fourteen studies divided the patients into two groups (GPS $1 / 2$ vs 0 ). The other four studies divided the patients into three groups (GPS $=0,1$ and 2), so the results of GPS 1 vs 0 and 2 vs 0 were obtained. Since the patients were divided into different categories, this meta-analysis will also be conducted from different groups. At the same time, the study by Sprinzl et $a l^{10}$ contained two cohorts, and the data were summarised from two independent treatment cohorts and reported separately: Sprinzl (V) and Sprinzl (E).

As shown in figure $3 \mathrm{~A}$, there was moderate heterogeneity in the GPS $1 / 2$ vs 0 group $\left(\mathrm{I}^{2}=59.4, \mathrm{p}=0.002\right)$, and a random-effect model was used. Patients with a GPS of 1 or 2 had poorer OS than those with a GPS of $0(p<0.001)$. When patients were divided into three groups, moderate heterogeneity was also detected and the random-effect model was used (GPS 1 vs $0, \mathrm{I}^{2}=72.1 \%, \mathrm{p}=0.006$, and GPS 2 vs $\left.0, \mathrm{I}^{2}=71.4 \%, \mathrm{p}=0.007\right)$. These results reveal that the level of GPS has a significant correlation with prognosis.

Moreover, mGPS used the same classification as GPS for calculation. There was low heterogeneity among these studies $\left(\mathrm{I}^{2}=36.1 \%, \mathrm{p}=0.166\right.$, and $\mathrm{I}^{2}=0.0 \%, \mathrm{p}=0.464$ for mGPS $1 / 2$ vs 0 and mGPS 2 vs 0 , respectively), and a fixedeffect model was used. These results reveal that elevated mGPS has a significant correlation with poor OS (both $\mathrm{p}<0.001)$. Moderate heterogeneity $\left(\mathrm{I}^{2}=60.1 \%, \mathrm{p}=0.057\right)$ was detected among mGPS 1 vs 0 , and the results showed that no significant difference was found between mGPS and OS.

\section{GPS/mGPS and DFS or RFS}

As shown in figure 3B, the results showed that the difference between GPS and DFS was not statistically significant (HR=1.69, 95\% CI 0.90 to 3.14). In addition, no correlation between mGPS and DFS was found ( $\mathrm{HR}=2.03,95 \%$ CI 0.94 to 4.38; figure 3B). Moreover, there was no relationship between GPS and RFS (HR=0.98, 95\% CI 0.61 to 1.57; figure 3C). Due to the small number of studies included, we believe that further experimental and theoretical studies are needed to verify this conclusion.

\section{Subgroup analyses}

Considering the moderate heterogeneity of the studies, subgroup analysis was conducted by the main treatment (surgical vs non-surgical), sample size ( $\geq 300$ vs $<300$ ), variable type (multivariate vs univariate), study design (retrospective vs prospective) and study region. The detailed results are summarised in table 2. Among the groups (GPS $1 / 2$ vs 0 ), a significant relationship between increased GPS and inferior OS was detected in all the aforementioned subgroups. According to the 


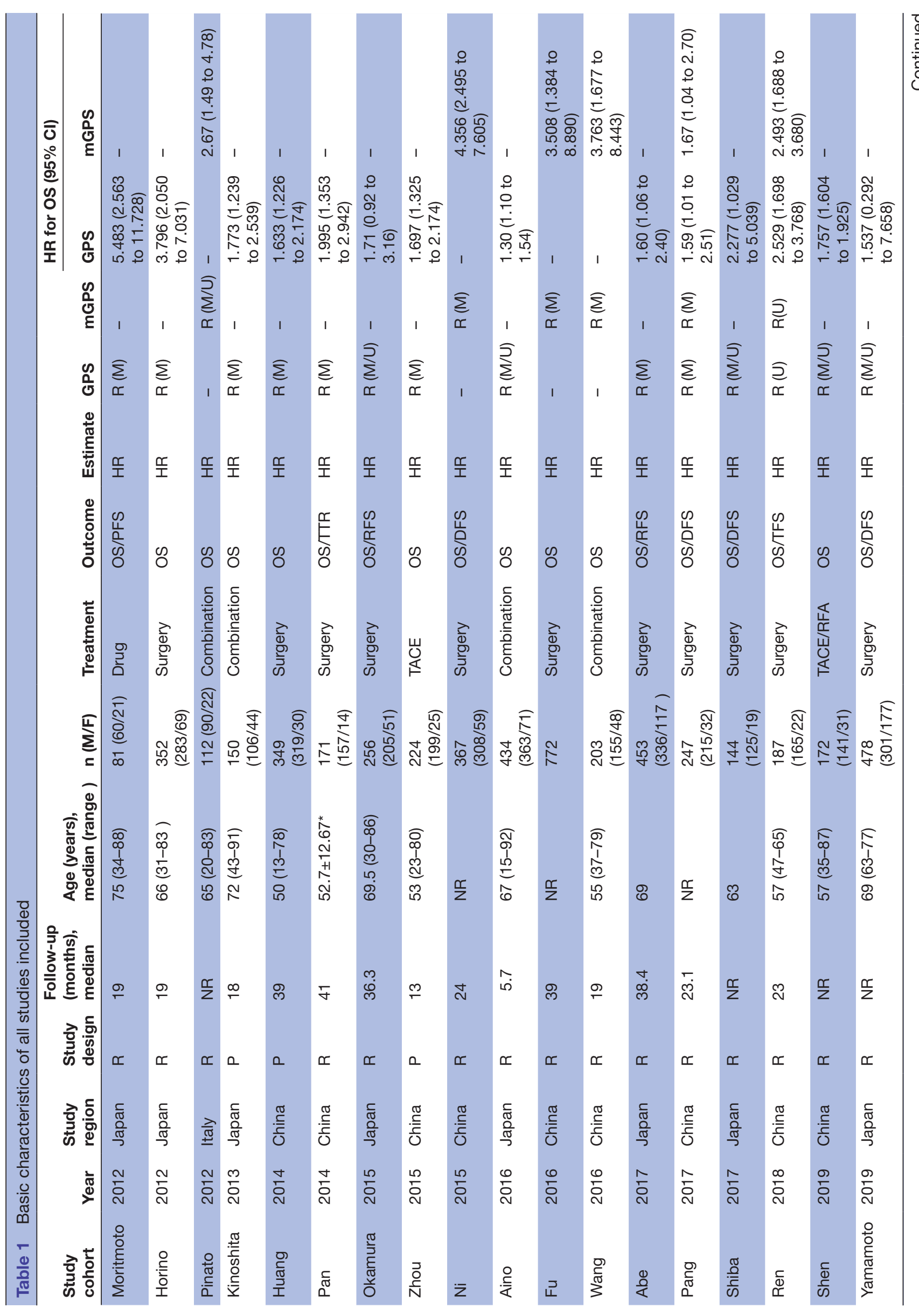




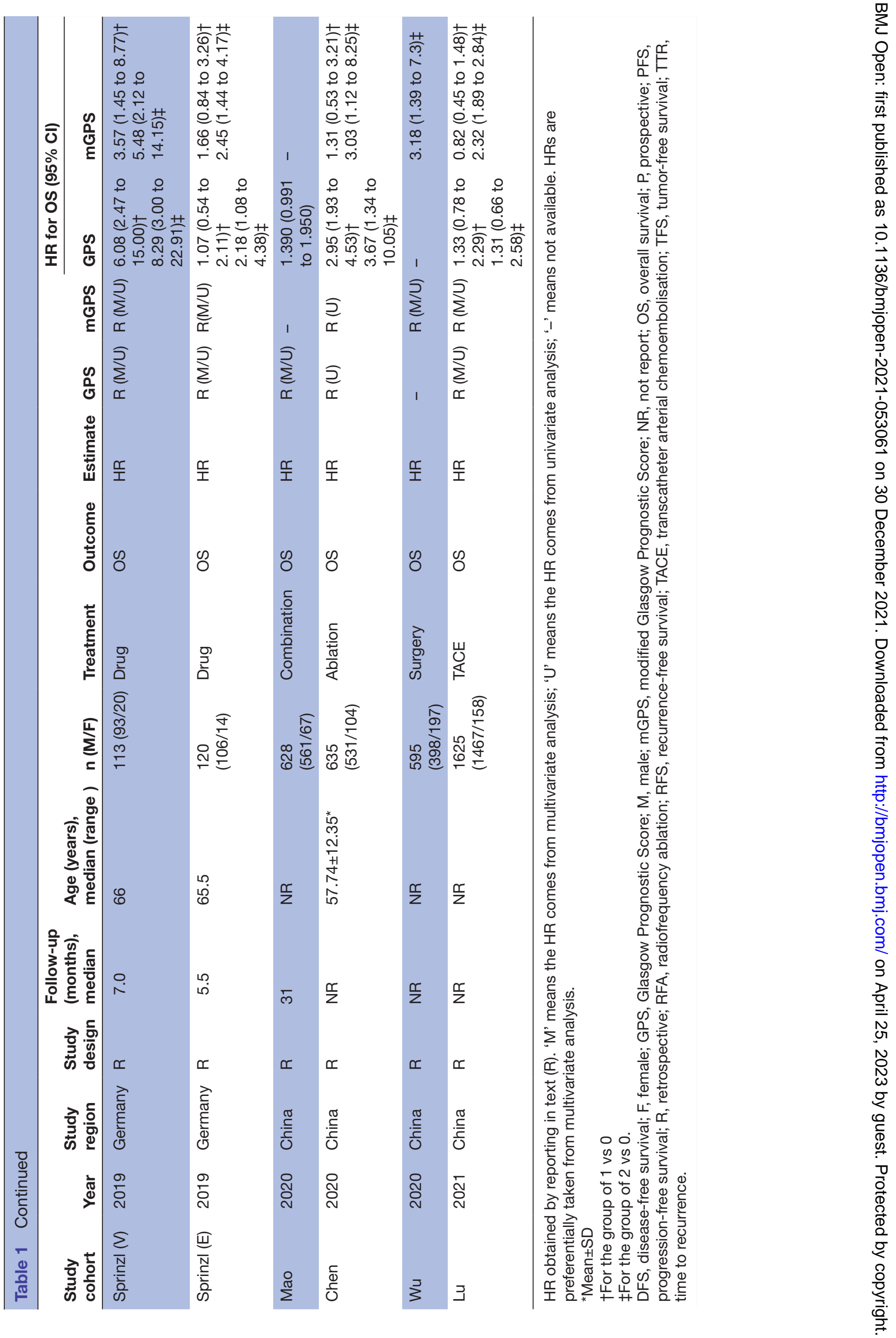




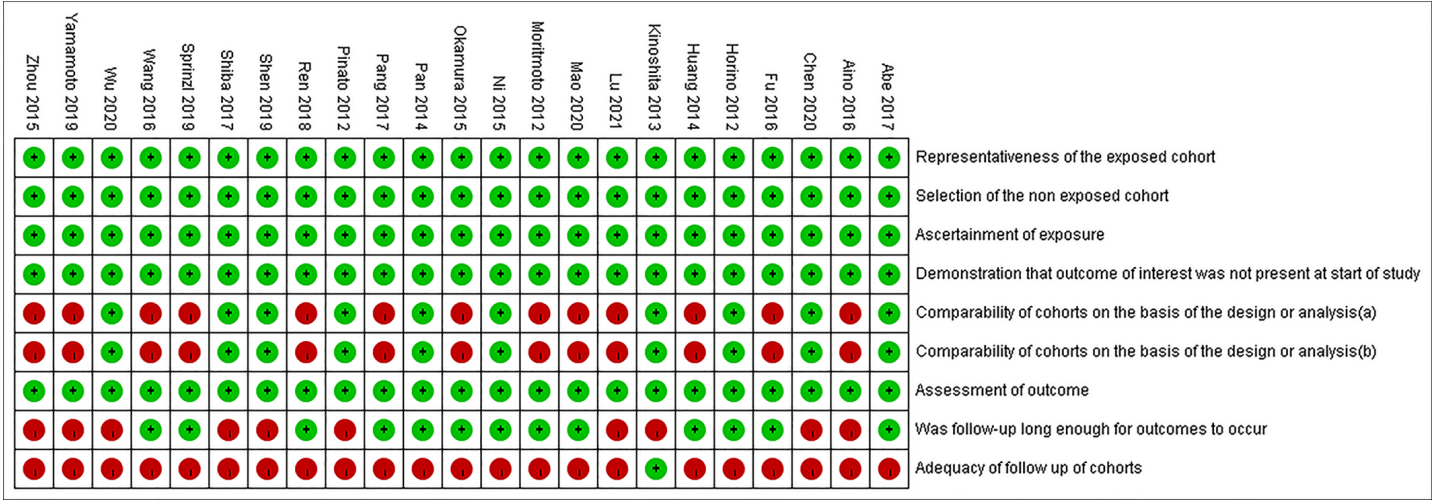

Figure 2 Risk of bias assessment for studies included in the review.

results of the subgroup analysis, there were significant differences in $\mathrm{I}^{2}$ values after grouping, and the source of heterogeneity was related to treatment methods, sample size, study design and region. As shown in table 2 , in the studies with GPS 1 vs 0 and that of 2 vs 0 , region-based subgroup analyses showed that patients with elevated GPS in China were not associated with decrease in OS (each $\mathrm{p}>0.05$ ). In addition, treatment-based subgroup analysis indicated that there was an association between GPS and OS.

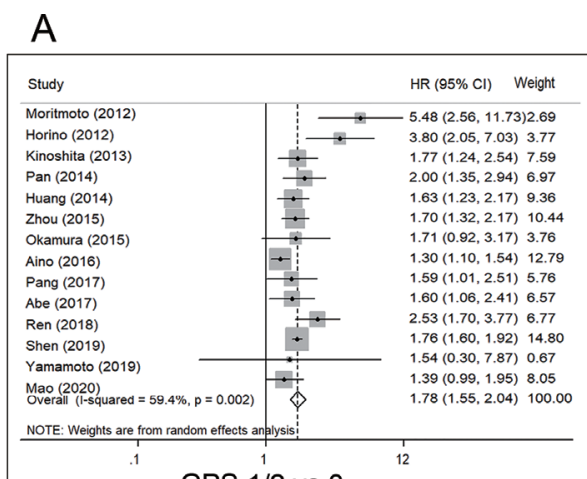

GPS $1 / 2$ vs 0
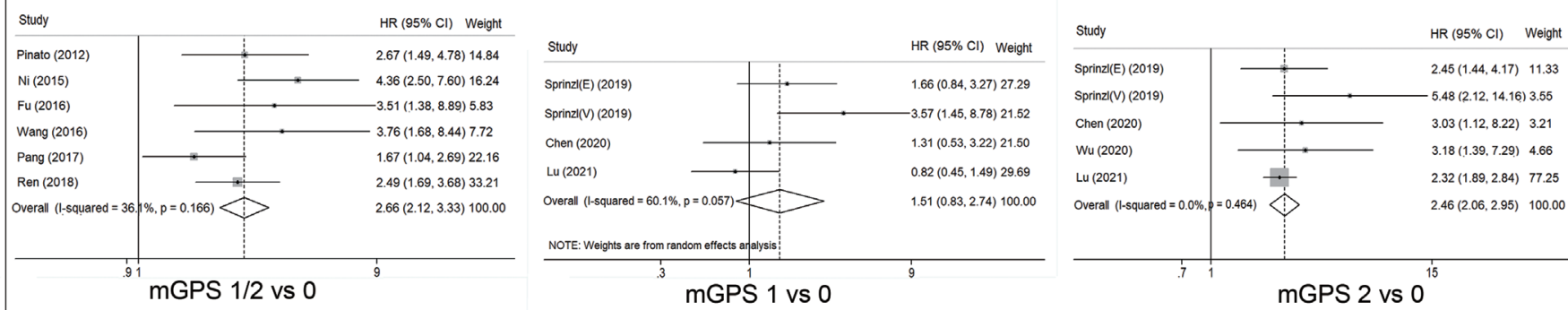

(OS) 
Table 2 Overall and subgroup meta-analyses of the relationship between GPS/mGPS and OS

\section{Fixed-effect model}

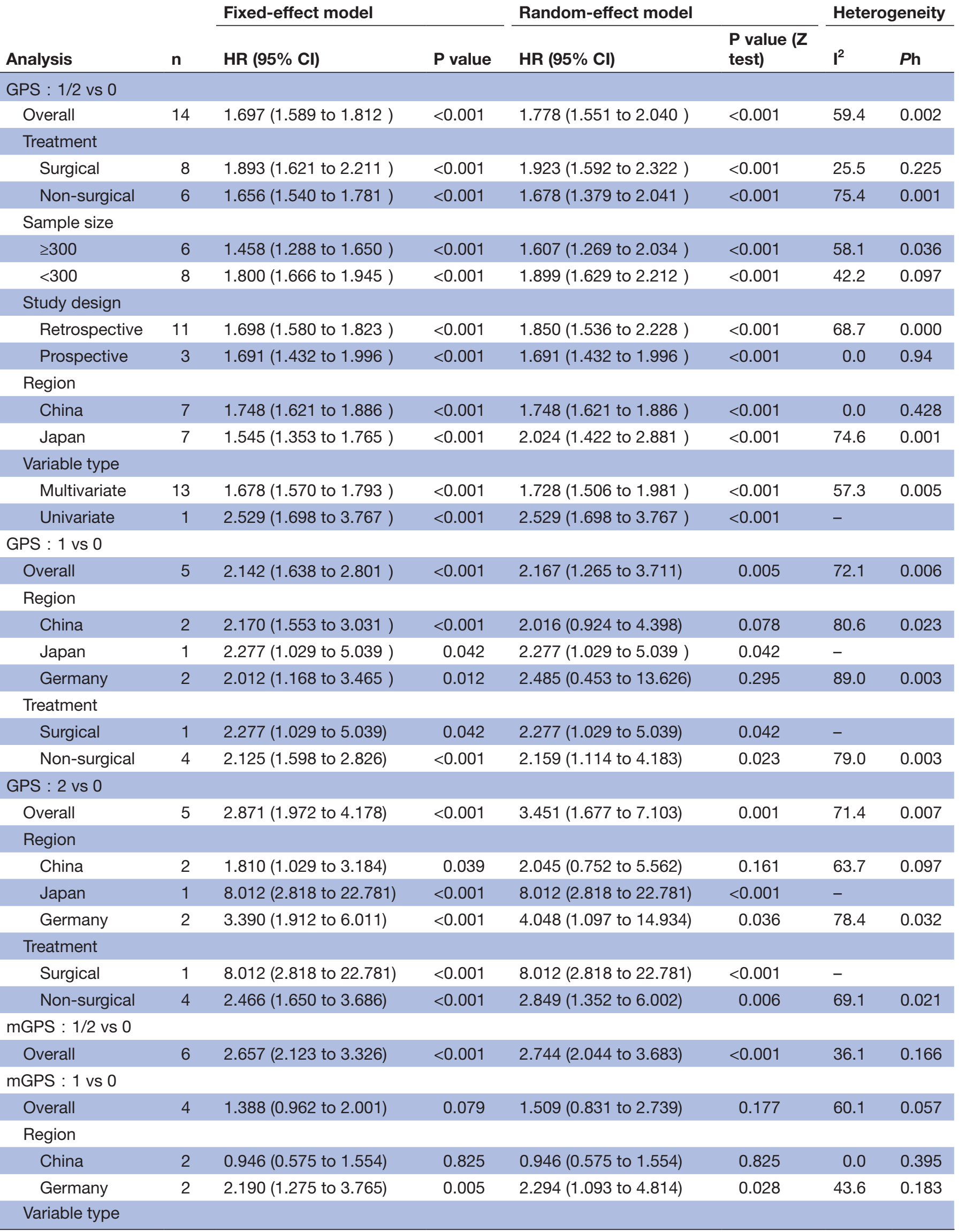


Table 2 Continued

\begin{tabular}{|c|c|c|c|c|c|c|c|}
\hline \multirow[b]{2}{*}{ Analysis } & \multirow[b]{2}{*}{$\mathbf{n}$} & \multicolumn{2}{|l|}{ Fixed-effect model } & \multicolumn{2}{|l|}{ Random-effect model } & \multicolumn{2}{|c|}{ Heterogeneity } \\
\hline & & HR $(95 \% \mathrm{Cl})$ & $P$ value & HR $(95 \% \mathrm{Cl})$ & $\begin{array}{l}P \text { value ( } Z \\
\text { test) }\end{array}$ & $\mathbf{I}^{2}$ & $P h$ \\
\hline Univariate & 1 & $1.310(0.532$ to 3.224$)$ & 0.557 & $1.310(0.532$ to 3.224$)$ & 0.557 & - & \\
\hline \multicolumn{8}{|l|}{ mGPS: 2 vs 0} \\
\hline
\end{tabular}

'-' means not available; ' $n$ ' means the number of studies

GPS, Glasgow Prognostic Score; mGPS, modified Glasgow Prognostic Score.

\section{Sensitivity analysis}

The effect on the combined effect size was assessed by excluding a particular study in isolation. The results revealed that the combined effect size was robust and no significant bias was found (figure 4).

\section{Publication bias}

Begg's funnel plot and Egger's test were used to evaluate the publication bias of the included studies (figure 5). For only GPS 2 vs 0 (figure 5A), publication bias was detected by Egger's test $(p=0.029)$. Using trim-and-fill analysis, the results showed that two studies did not evaluate the prognostic role of GPS in the OS of HCC (figure 5B). Analysis results of other groups indicated that the funnel plot was symmetric and Begg's test linear regression test showed no significant publication bias.

\section{DISCUSSION}

Increasing evidence proved that the long-term survival for patients with tumours is significantly associated with the level of tumour-associated inflammation. ${ }^{35}$ In addition to mGPS and GPS, there are many inflammation-based prognostic markers to predict survival and prognosis of HCC, including prognostic index (PI), prognostic nutritional index (PNI), neutrophil-to-lymphocyte ratio (NLR), platelet-to-lymphocyte ratio (PLR), systemic immuneinflammation index (SII) and lymphocyte-monocyte ratio (LMR) ${ }^{36}$ These inflammatory indicators have a complex relationship with survival and recurrence of HCC. Therefore, it is important to identify non-invasive and readily available pretreatment variables to assess the survival outcomes in HCC. Several studies have investigated the
A

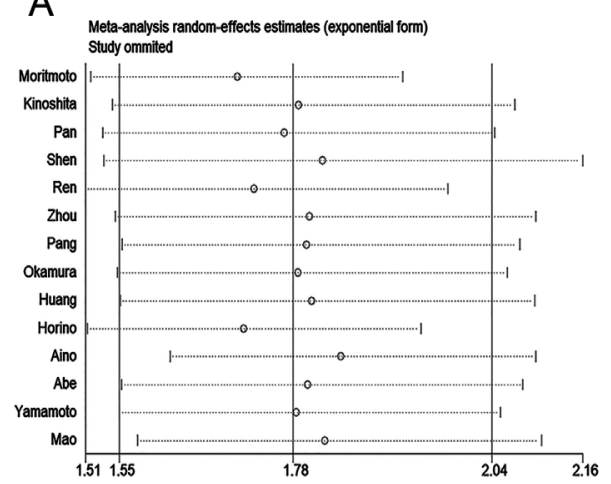

D

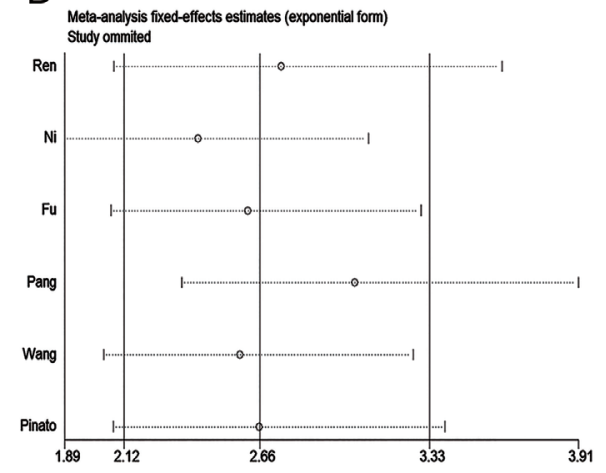

B

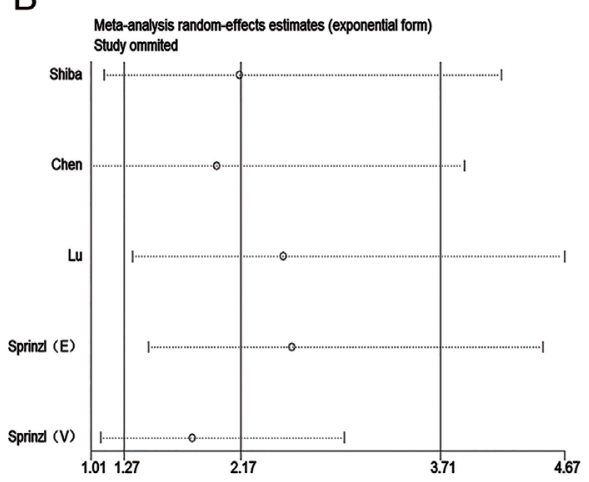

E

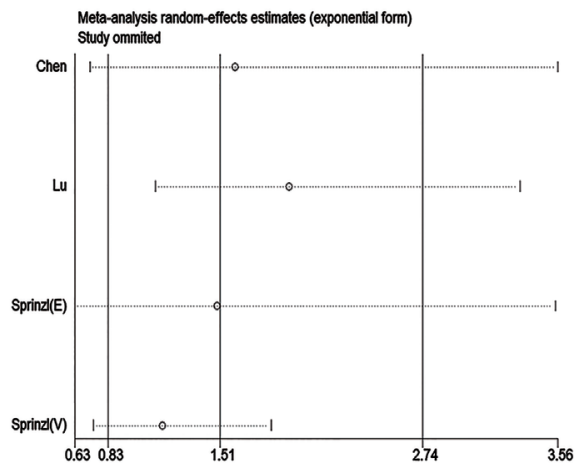

C

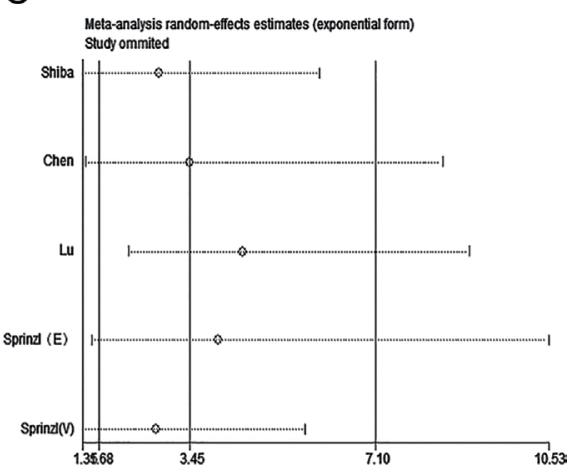

F

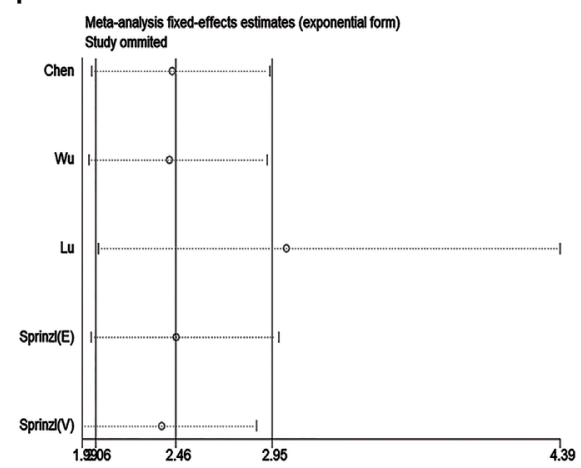

Figure 4 Influence analysis of studies enrolled in the present meta-analysis. (A) GPS 1/2 vs 0, (B) GPS 1 vs 0 , (C) GPS 2 vs 0 , (D) mGPS $1 / 2$ vs 0, (E) mGPS 1 vs 0 and (F) mGPS 2 vs 0. GPS, Glasgow Prognostic Score; mGPS, modified Glasgow Prognostic Score. 

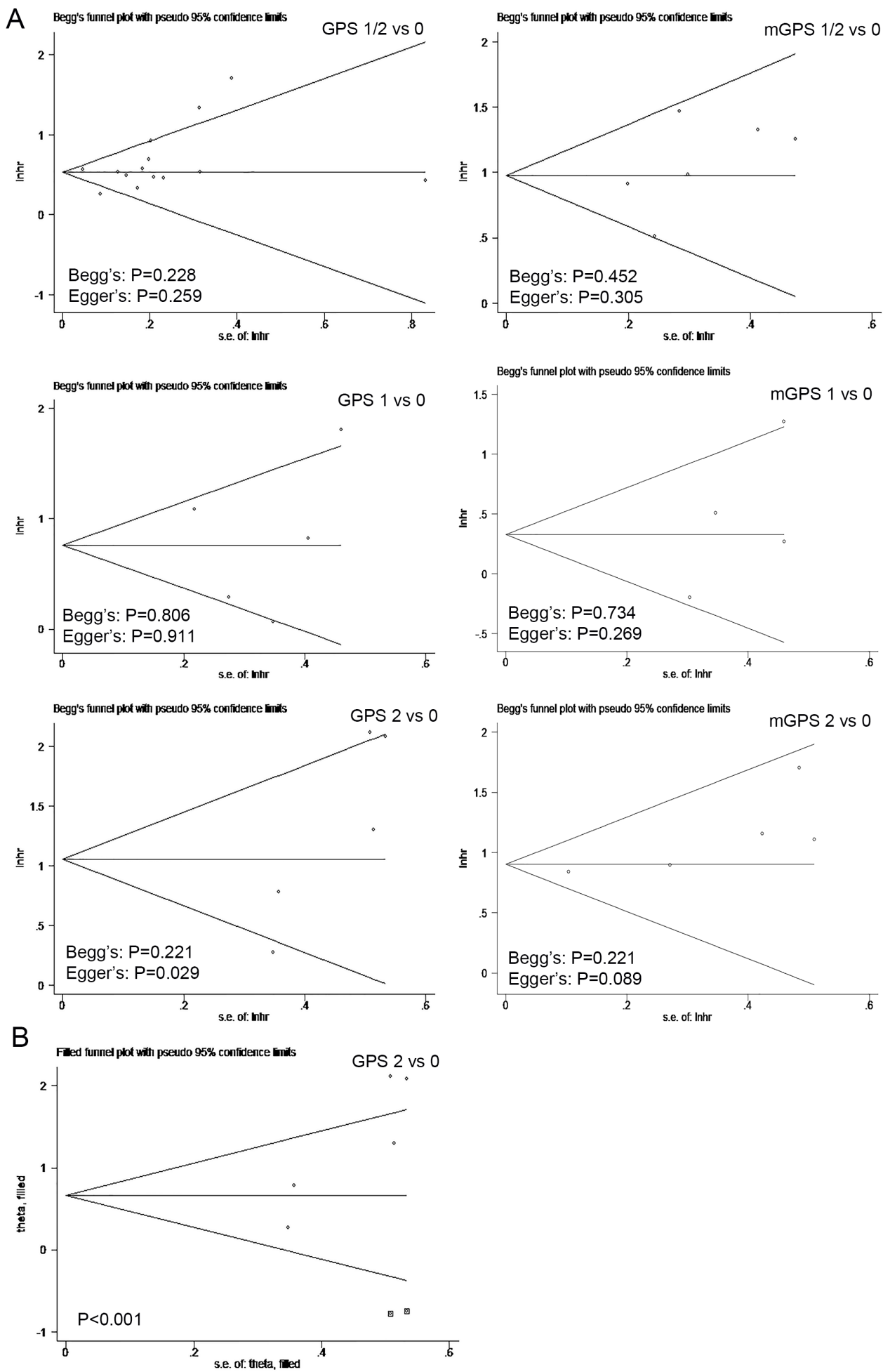

Figure 5 Funnel plot for publication bias. (A) The Begg's funnel plot. (B) Trim-and-fill analysis: GPS 2 vs 0. GPS, Glasgow Prognostic Score; mGPS, modified Glasgow Prognostic Score.

association between pretreated GPS/mGPS levels and survival in patients with HCC, but the results were inconsistent. Meanwhile, both mGPS and GPS are scored on the basis of CRP and albumin, and the scoring criteria are very similar. There is no consensus on which is more suitable for clinical use. Then we reviewed published studies and performed a meta-analysis to obtain a scientific theoretical basis for evaluating the association between pretreatment GPS/mGPS and survival in patients with HCG.

Most studies have shown that higher GPS was associated with increased $\alpha$-fetoprotein, larger tumour diameter, vascular invasion, extrahepatic metastasis, higher ChildPugh grade and higher scores of the Cancer of the Liver Italian Program. ${ }^{18}{ }^{21}$ In addition, GPS and mGPS were related to postrecurrence survival in patients with early 
recurrent HCC. ${ }^{36}$ However, multivariate analysis of some studies $^{2125}$ showed that GPS is not associated with survival in patients with HCC. Previous meta-analysis ${ }^{37}$ has found that GPS was associated with survival outcomes in HCC. However, HR for one of the included studies was estimated rather than obtained from text reports, and the studies did not use the same cut-off values. This aspect needs to be further explored. Therefore, we expanded the sample size, eliminated the studies that could not clearly give HRs and then conducted grouping studies according to cutoff values. At the same time, we further explored whether mGPS or GPS is more suitable as a biomarker for the survival prognosis of HCC. Our meta-analysis combined the outcomes from 23 individual studies, suggesting that elevated GPS was strongly associated with poorer OS in patients with HCC regardless of which subgroup. This also filled the gap in the previous meta-analysis. Furthermore, no correlation was found between GPS and DFS or RFS. In addition, mGPS was also associated with the prognosis of HCC. However, some of the variables did not show an association with OS, for example, an mGPS of 1 vs 0 . Therefore, compared with the mGPS, GPS is more closely associated with the survival in patients with HCC.

The exact mechanism between tumour and inflammation in those patients with cancer is still undefined. However, accumulating evidence has indicated that the Inflammation-related indicators is a reliable predictor of tumour-specific survival. The level of circulating immune cells is associated with tumour-associated inflammation. ${ }^{34}$ Lymphocytes play an important antitumour role, and when lymphocytes are damaged, they may lead to the spread of tumour cells. Elevated CRP also suggests damage to T lymphocyte. Meanwhile, CRP was an acutephase protein produced by liver cells and regulated by inflammatory cytokines. Inflammatory cytokines promote angiogenesis and inhibit host immune responses when tumours develop or invade. ${ }^{38}{ }^{39}$ Hypoalbuminaemia reflects poor liver function synthesis and may be caused by chronic inflammation. Albumin also reflects the nutritional status of the patient. As albumin decreases, the body's status decreases, increasing mortality.

There are many scores or indexes related to these two inflammatory indicators, including mGPS, GPS, PI, PNI, LCR and CAR. ${ }^{11} 2129$ Among these scores, mGPS and GPS were superior to other inflammation-based scores as prognostic indicators, such as PI and PNI, in the prognosis of patients with liver cancer after surgical treatment. ${ }^{17} 2128$ $\mathrm{Ni}$ et al reported that mGPS was better than GPS in using as an independent prognostic indicator of postoperative liver cancer, ${ }^{21}$ but other studies have come to the opposite conclusion. ${ }^{19}$ In addition, mGPS has been shown to be more closely associated with the prognosis of patients with hepatitis B virus-related HCC. ${ }^{26}$ GPS was superior to mGPS as a prognostic factor in evaluating the prognosis of patients with HCC with different disease stages and liver function states. ${ }^{18}$ GPS, mGPS and PNI have been reported as predictors of early recurrence after hepatectomy. ${ }^{36}$ For patients with HCC treated with TACE, LCR has better prognostic ability than PNI, mGPS and GPS, ${ }^{11}$ which in turn have better prognostic ability than PNI and mGPS ${ }^{23}$ Therefore, mGPS/GPS played an important role in the prognosis of HCC. However, it is not clear which indicator is more suitable for clinical application. So, we explored this issue and found that GPS is more suitable for assessing the survival prognosis of HCC than mGPS.

There are still a few limitations to this meta-analysis. First, in the included research results, the cut-off value of GPS/mGPS was different. Although we have conducted classification analysis and discussion, the sample size has been virtually reduced, which will inevitably have a potential impact. Second, although most of the HRs included were derived from multivariable analyses, there is no guarantee that the same set of covariables was used during the analyses, which may have influenced the results. Third, a major limitation of this study was that most of the included studies were retrospective in design, which may lead to selection bias. Fourth, the NOS score of four articles with published data was 5, which was not of very high quality. Moreover, some baseline characteristics are different, such as age, Barcelona Clinic liver Cancer (BCLC) stage and duration of follow-up, which may cause heterogeneity. In addition, this study was limited to research published in English and may lead to language-induced selection bias. Hence, underlying heterogeneity is inevitable. In our study, random-effect or fixed-effect models were used according to the heterogeneity. However, heterogeneity was affected by many aspects, and there was some uncertainty in comparing the results of different effect models.

In conclusion, this study showed that pretreatment mGPS or GPS was associated with OS in patients with HCC, which could be used as one of the effective biomarkers. It can be combined with other indicators, such as tumour stage or pathological stage, to predict prognosis. Compared with the mGPS, GPS was more closely associated with the survival in patients with HCC. However, a large randomised controlled trial is needed to verify our findings, to explore the complex relationship between the microenvironment of HCC and the inflammatory response, and to clarify the mechanism of these inflammatory markers in HCC.

Contributors $\mathrm{LL}, \mathrm{KL}$ and $\mathrm{DL}$ : conceptualisation and writing-original draft. $\mathrm{LL}$ and $\mathrm{KL}$ : investigation, methodology, resources and investigation. HW and JZ: formal analysis. All authors: writing, review and editing. LL is the author responsible for the overall content of this study.

Funding This study was supported by grants from the Natural Science Foundation of Fujian Province (grant number: 2016J01590) and the 900th Hospital of the Joint Logistics Support Force Fund (grant number:2020Z12).

Competing interests None declared.

Patient consent for publication Not applicable.

Ethics approval This study does not involve human participants.

Provenance and peer review Not commissioned; externally peer reviewed.

Data availability statement All data relevant to the study are included in the article or uploaded as supplementary information. All literature related to this study is included in the article, and the data for the meta-analysis are included in the manuscript. 
Supplemental material This content has been supplied by the author(s). It has not been vetted by BMJ Publishing Group Limited (BMJ) and may not have been peer-reviewed. Any opinions or recommendations discussed are solely those of the author(s) and are not endorsed by BMJ. BMJ disclaims all liability and responsibility arising from any reliance placed on the content. Where the content includes any translated material, BMJ does not warrant the accuracy and reliability of the translations (including but not limited to local regulations, clinical guidelines, terminology, drug names and drug dosages), and is not responsible for any error and/or omissions arising from translation and adaptation or otherwise.

Open access This is an open access article distributed in accordance with the Creative Commons Attribution Non Commercial (CC BY-NC 4.0) license, which permits others to distribute, remix, adapt, build upon this work non-commercially, and license their derivative works on different terms, provided the original work is properly cited, appropriate credit is given, any changes made indicated, and the use is non-commercial. See: http://creativecommons.org/licenses/by-nc/4.0/.

ORCID iD

Dongliang Li http://orcid.org/0000-0001-5536-8468

\section{REFERENCES}

1 Torre LA, Bray F, Siegel RL, et al. Global cancer statistics, 2012. CA Cancer J Clin 2015;65:87-108.

2 Poon RTP. Prevention of recurrence after resection of hepatocellular carcinoma: a daunting challenge. Hepatology 2011;54:757-9.

3 Forrest LM, McMillan DC, McArdle CS, et al. Evaluation of cumulative prognostic scores based on the systemic inflammatory response in patients with inoperable non-small-cell lung cancer. Br J Cancer 2003;89:1028-30.

4 Ishizuka M, Nagata H, Takagi K, et al. Inflammation-Based prognostic score is a novel predictor of postoperative outcome in patients with colorectal cancer. Ann Surg 2007;246:1047-51.

5 Nozoe T, Iguchi T, Egashira A, et al. Significance of modified Glasgow prognostic score as a useful indicator for prognosis of patients with gastric carcinoma. Am J Surg 2011;201:186-91.

6 Vashist YK, Loos J, Dedow J, et al. Glasgow prognostic score is a predictor of perioperative and long-term outcome in patients with only surgically treated esophageal cancer. Ann Surg Oncol 2011;18:1130-8.

7 Proctor MJ, Morrison DS, Talwar D, et al. An inflammation-based prognostic score (mGPS) predicts cancer survival independent of tumour site: a Glasgow inflammation outcome study. Br J Cancer 2011;104:726-34.

8 Okano N, Kasuga A, Kawai K, et al. The modified Glasgow prognostic score in patients with gemcitabine-refractory biliary tract cancer. Anticancer Res 2018;38:1755-61.

9 Pang S, Zhou Z, Yu X, et al. The predictive value of integrated inflammation scores in the survival of patients with resected hepatocellular carcinoma: a retrospective cohort study. Int J Surg 2017;42:170-7.

10 Sprinzl MF, Kirstein MM, Koch S, et al. Improved prediction of survival by a risk Factor-Integrating inflammatory score in SorafenibTreated hepatocellular carcinoma. Liver Cancer 2019;8:387-402.

11 Lu L-H, Wei W, Li S-H, et al. The lymphocyte-C-reactive protein ratio as the optimal inflammation-based score in patients with hepatocellular carcinoma underwent TACE. Aging 2021;13:5358-68.

12 Moher D, Liberati A, Tetzlaff J, et al. Preferred reporting items for systematic reviews and meta-analyses: the PRISMA statement. PLoS Med 2009;6:e1000097.

13 Stroup DF, Berlin JA, Morton SC, et al. Meta-Analysis of observational studies in epidemiology: a proposal for reporting. meta-analysis of observational studies in epidemiology (moose) group. JAMA 2000;283:2008-12.

14 Higgins JPT, Thompson S, Deeks J. Measuring inconsistency in meta-analyses. BMJ 2003;327:557-60.

15 Morimoto M, Numata K, Moriya S, et al. Inflammation-Based prognostic score for hepatocellular carcinoma patients on sorafenib treatment. Anticancer Res 2012;32:619-23.

16 Pinato DJ, Stebbing J, Ishizuka M, et al. A novel and validated prognostic index in hepatocellular carcinoma: the inflammation based index (IBI). J Hepatol 2012;57:1013-20.
17 Horino K, Beppu T, Kuroki H, et al. Glasgow prognostic score as a useful prognostic factor after hepatectomy for hepatocellular carcinoma. Int J Clin Oncol 2013;18:829-38.

18 Kinoshita A, Onoda H, Imai N, et al. The Glasgow prognostic score, an inflammation based prognostic score, predicts survival in patients with hepatocellular carcinoma. BMC Cancer 2013;13:52.

19 Huang J, Xu L, Luo Y, et al. The inflammation-based scores to predict prognosis of patients with hepatocellular carcinoma after hepatectomy. Med Oncol 2014;31:883.

20 Pan Q-X, Zhang J-H, Su Z-J, et al. The Glasgow prognostic score is an independent prognostic predictor of hepatocellular carcinoma following radical resection. Oncol Res Treat 2014;37:192-7.

$21 \mathrm{Ni} \mathrm{X-C,} \mathrm{Yi} \mathrm{Y,} \mathrm{Fu} \mathrm{Y-P,} \mathrm{XC} \mathrm{N,} \mathrm{YP} \mathrm{F,} \mathrm{et} \mathrm{al.} \mathrm{Prognostic} \mathrm{value} \mathrm{of} \mathrm{the}$ modified Glasgow prognostic score in patients undergoing radical surgery for hepatocellular carcinoma. Medicine 2015;94:e1486.

22 Okamura Y, Ashida R, Ito T, et al. Preoperative neutrophil to lymphocyte ratio and prognostic nutritional index predict overall survival after hepatectomy for hepatocellular carcinoma. World $J$ Surg 2015;39:1501-9.

23 Zhou D-S, Xu L, Luo Y-L, et al. Inflammation scores predict survival for hepatitis $B$ virus-related hepatocellular carcinoma patients after transarterial chemoembolization. World $\mathrm{J}$ Gastroenterol 2015;21:5582-90.

24 Aino $\mathrm{H}$, Sumie S, Niizeki T, et al. The systemic inflammatory response as a prognostic factor for advanced hepatocellular carcinoma with extrahepatic metastasis. Mol Clin Oncol 2016;5:83-8.

$25 \mathrm{Fu}$ Y-P, Ni X-C, Yi Y, et al. A novel and validated inflammation-based score (IBS) predicts survival in patients with hepatocellular carcinoma following curative surgical resection: a STROBE-Compliant article. Medicine 2016;95:e2784.

26 Wang JQ, Cheng PG, MY L. Comparison of the prognostic value of C-reactive protein-based prognostic scores in patients with hepatitis $B$ virus-related hepatocellular carcinoma. International Journal of Clinical and Experimental Medicine 2016;9:8272-9.

27 Abe T, Tashiro H, Kobayashi T, et al. Glasgow prognostic score and prognosis after hepatectomy for hepatocellular carcinoma. World $J$ Surg 2017;41:1860-70.

28 Shiba H, Horiuchi T, Sakamoto T, et al. Glasgow prognostic score predicts therapeutic outcome after hepatic resection for hepatocellular carcinoma. Oncol Lett 2017:14:293-8.

29 Ren Y, Fan X, Chen G, et al. Preoperative C-reactive protein/ albumin ratio to predict mortality and recurrence of patients with hepatocellular carcinoma after curative resection. Med Clin 2019;153:183-90.

30 Shen Y, Wang H, Li W, et al. Prognostic significance of the CRP/ $\mathrm{Alb}$ and neutrophil to lymphocyte ratios in hepatocellular carcinoma patients undergoing TACE and RFA. J Clin Lab Anal 2019;33:e22999.

31 Yamamoto M, Kobayashi T, Kuroda S, et al. Verification of inflammation-based prognostic marker as a prognostic indicator in hepatocellular carcinoma. Ann Gastroenterol Surg 2019;3:667-75.

32 Chen S, Ma W, Cao F, et al. Hepatocellular carcinoma within the Milan criteria: a novel inflammation-based nomogram system to assess the outcomes of ablation. Front Oncol 2020;10:10.

33 Mao M, Wang X, Song Y, et al. Novel prognostic scores based on plasma prothrombin time and fibrinogen levels in patients with AFP-Negative hepatocellular carcinoma. Cancer Control 2020;27:107327482091552.

34 Wu X, Sun Z, Zhu Y. Prognostic effect of systemic inflammation in patients undergoing surgery for hepatocellular carcinoma: comparison of composite ratios and cumulative scores. Transl Cancer Res 2020;9:6154-65.

35 Grivennikov SI, Greten FR, Karin M. Immunity, inflammation, and cancer. Cell 2010;140:883-99.

36 Wang C, He W, Yuan Y, et al. Comparison of the prognostic value of inflammation-based scores in early recurrent hepatocellular carcinoma after hepatectomy. Liver Int 2020;40:229-39.

$37 \mathrm{Li} \mathrm{M-X,} \mathrm{Bi} \mathrm{X-Y,} \mathrm{Li} \mathrm{Z-Y,} \mathrm{et} \mathrm{al.} \mathrm{Prognostic} \mathrm{role} \mathrm{of} \mathrm{Glasgow} \mathrm{prognostic}$ score in patients with hepatocellular carcinoma: a systematic review and meta-analysis. Medicine 2015;94:e2133.

38 Gómez-Gómez E, Carrasco-Valiente J, Campos-Hernández JP, et al. Clinical association of metabolic syndrome, C-reactive protein and testosterone levels with clinically significant prostate cancer. J Cell Mol Med 2019;23:934-42.

39 Mantovani A, Allavena P, Sica A, et al. Cancer-Related inflammation. Nature 2008;454:436-44. 